\title{
Objective Empirical Mode Decomposition metric
}

\author{
Dawid Laszuk, Oswaldo Cadenas, Sławomir J Nasuto
}

\begin{abstract}
Empirical Mode Decomposition (EMD) is a datadriven technique for extraction of oscillatory components from data. Although it has been introduced over 15 years ago, its mathematical foundations are still missing which also implies lack of objective metrics for decomposed set evaluation. Currently, the most common technique for assessing results of EMD is their visual inspection, which is very subjective. This article provides objective measures for validating EMD output, which are derived from the original definition of oscillatory components. Three proposed metrics refer to component's idealised characteristics, i.e. its significant instantaneous frequency and the ability to extract amplitude- and frequency-modulated parts. Possible application of these metrics is presented on two examples.
\end{abstract}

Keywords-Empirical Mode Decomposition, Hilbert-Huang Transform, Hilbert Transform, Intrinsic Mode Function, Metric, Signal Decomposition.

\section{INTRODUCTION}

Empirical mode decomposition (EMD) has been proposed recently by Huang et al. [1]. Over past 15 years the method has gained worldwide recognition as a data-driven method for extraction of physically meaningful oscillatory components [2]-[4].

In the original paper on EMD [1] authors noted that small perturbations to the input signal results in different outputs. This is highly undesired effect, but unfortunately due to the heuristic nature of the EMD it is impossible to determine which set of intrinsic mode functions (IMFs) - the output of the EMD - is better. There were many attempts to improve the algorithm, for example ensemble empirical mode decomposition [5]. It assumes that adding small fluctuations to the input signal results in slight perturbation of the output around true decomposition. Thus, creating large ensemble of signals with added small noise will exhaust all possible perturbations and result in large ensemble of decompositions with the mean value being the true set. However, evaluations of this approach indicate that obtained results most of the time do not resemble oscillations and the problem of method being empirical remains. Other attempts to improve EMD, e.g. [6]-[8], have similar issues; there is no mathematical framework and all manipulations are driven mainly with intuition. Nevertheless, researchers usually can make, and do, assessments of method's performance based on their knowledge and experience. Few authors ( [1], [9], [10]) have made attempts to formulate rules based on which sets of IMFs should be chosen. All of them, however, are based on the assumption that IMFs belong to Hilbert's $L^{2}$ function space, which is not necessarily true. As stated in [1] obtained orthogonality (in Hilbert sense) is purely by coincidence and should not be expected from the method.

Manuscript received November 16, 2015, revised January 13, 2016.

The authors are with the School of Systems Engineering, University of Reading, Reading, RG6 6AY, UK (e-mail: d.laszuk@pgr.reading.ac.uk).
The most popular validation method is based on a visual inspection of the results [9], [11], [12]. This introduces the artefact of subjective opinion into the process. Conclusions from their research cannot be generalized, since they have looked at different, often single, features of output. In this article objective methods for validating the decomposed sets are introduced. The proposals are based on the features that IMF are expected to possess. Each variant focuses on a different characteristics of the data.

The rest of the paper is organized as follows. Section II introduces empirical mode decomposition. Section III describes proposed validating methods. Section IV describes two conducted numerical experiments with conclusions in section V.

\section{EMPIRICAL MODE DECOMPOSITION}

Empirical mode decomposition (EMD) is a data-driven method for time-frequency analysis. It decomposes data into components called intrinsic mode functions (IMFs). The algorithm encapsulating performing EMD on signal $S(t)$ is as follows:

1) Assign trend as a difference between input signal and sum of extracted components, i.e. $r_{0}(t):=S(t)-$ $\sum_{j=1}^{n} c_{j}(t)$. If it is a first iteration, than $r_{0}(t):=S(t)$.

2) Identify all local extrema (both minima and maxima) in time series $r_{i}(t)$, that is whenever the derivative is $d r_{i}(t) / d t=0$.

3) If the number of extrema is less or equal 2 then $r_{i}(t)$ is considered as a trend - a low frequency modulation - and the algorithm stops.

4) Calculate top $\left(e_{\max }\right)$ and bottom $\left(e_{\min }\right)$ envelopes by interpolating respectively local maxima and local minima with natural cubic splines.

5) Calculate the instantaneous mean of both envelopes $m(t)=\frac{1}{2}\left(e_{\max }(t)+e_{\min }(t)\right)$.

6) Subtract the mean from the input time series $h_{i}(t)=$ $r_{i}(t)-m_{i}(t)$. This step is called sifting, because it removes trend from fast varying component.

7) If $h_{i}(t)$ fulfills the stopping criteria, then it is considered to be an IMF (component $c_{n}(t):=h_{i}(t)$ ) and start again from step 1 with updated set of components. Otherwise, assigned residue as $r_{i+1}(t):=h_{i}(t)$ and repeat from step 2.

As a result, EMD decomposes signal $S(t)$ into a set of $N$ oscillatory $c_{j}(t)$ components (IMFs) and a trend $r(t)$ function. Procedure to perform EMD is not uniquely defined. It depends on interpolation technique used for spanning envelopes, definition of extrema, stopping criteria, etc. Different combinations of those settings can produce different outputs even for the same input data. This leads to an obvious question: which set of settings does produce the most significant results? 
In the original article on the EMD [1], it was suggested to apply the Hilbert transform (HT) to the obtained set of IMFs. The HT converts a real-valued signal $S(t)$ into an analytic form $A(t)$, which then can be decomposed into amplitude and phase modulation parts [13]. Mathematically, it can be presented as

$$
A(t)=a(t) \cos (\phi(t))
$$

where functions $a(t)$ and $\phi(t)$ are amplitude and phase, respectively. Taking derivative of phase over time $(\dot{\phi})$ allows to extract component's instantaneous frequency. With this terms average frequency can be defined as

$$
\dot{\Phi}=\frac{1}{T} \int_{0}^{T} \dot{\phi}(t) d t,
$$

where $T$ is the length of time over which the signal is observed. A combination of EMD and HT on all components is called Hilbert-Huang transformation.

\section{PROPOSED VALIDATION METHODS}

The main reason for method proposed in this paper is to avoid the use of subjective judgment in evaluation of EMD performance. This is achieved by relaying on the intrinsic features reportedly possessed by the IMFs, or on those that would help in the future analysis [1]. The main considered characteristics are: 1) decrease of average frequency with increase of IMFs index, 2) distinct instantaneous frequency for each IMF and 3) disjoint Fourier spectra support for IMF's amplitude and phase.

In this section, IMFs are represented in polar form, i.e. time series of the $j^{\text {th }}$ indexed IMF are assumed to have amplitude, $a$, and phase, $\phi$, modulations $\left(\mathrm{IMF}_{j}(t)=a_{j}(t) \cos \left(\phi_{j}(t)\right)\right.$. Although, all proposed metrics are designed for continuous functions, in most cases, change to the discrete domain is a straightforward operation. Such processing requires exchanging integration operator over time period $T$ into sum over all data points $P$.

\section{A. Validation method I}

This metric is based on the empirical evidence for the decrease of average instantaneous frequency, simply referred to as the average frequency, with the increase of IMF's index number. Although the order with which the IMF components are construced in general corresponds to the decreasing IMF average frequencies, there are instances when the instantaneous frequencies cross over between the components. Since it has been claimed that each IMF has a significant instantaneous frequency [1], such behaviour is unwelcome and hence it is penalised by this metric. Penalties are introduced when instantaneous frequency of an IMF with lower number (high average frequency) is smaller than instantaneous frequency of any IMF with higher number. The penalty value is proportional to the length of the crossing over effect, i.e.

$$
m_{j}^{\mathrm{I}}=\sum_{k=j+1}^{N} \int_{\dot{\phi}_{k}>\dot{\phi}_{j}} \frac{d t}{T}
$$

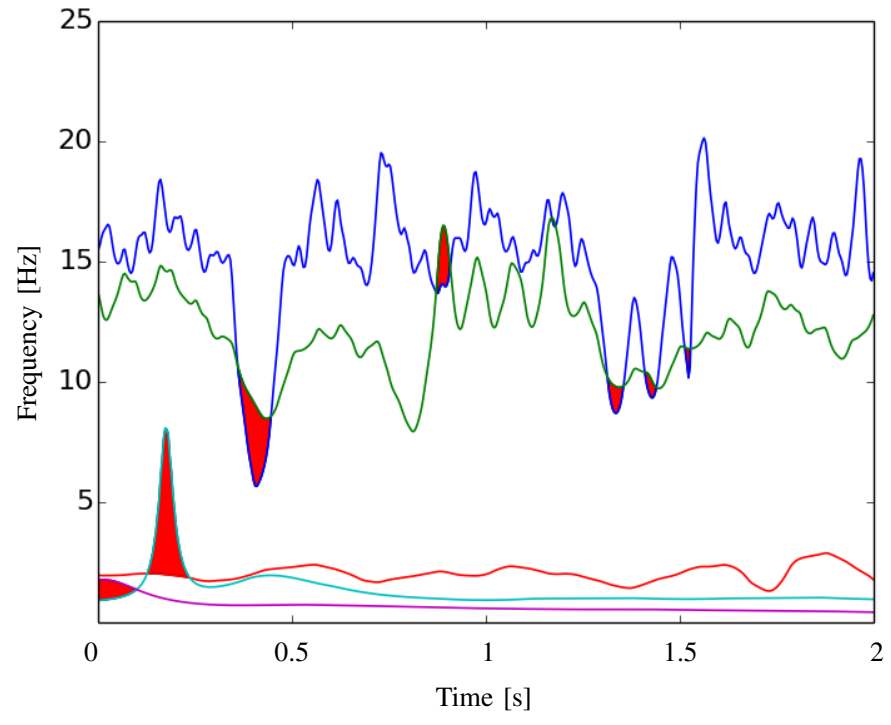

Fig. 1. Plot of instantaneous frequency for each IMF as a function of time Coloured regions indicates where the frequency crossing over occurs. Metric $M_{\mathrm{I}}$ penalises based on the length of those regions.

where $k, j$ are IMFs' indices. Formula (3) compares functions of instantaneous frequencies of two IMFs and returns the total duration, over which the IMF with higher index has lower frequency. The crossing over effect has been presented in Figure 1. It shows instantaneous frequency of each IMF as a function of time. Coloured regions indicate where the crossing over occurred. Summing over all pairs of IMFs allows us to assess results for particular EMD. Metric value for the whole set is given as

$$
M_{\mathrm{I}}=\sum_{j=1}^{N} m_{j}^{\mathrm{I}}, \quad M_{\mathrm{I}} \in\left[0, \frac{N(N-1)}{2}\right],
$$

According to this measure, the best IMF set is the one, for which $M_{\mathrm{I}}=0$, i.e. there are no crossing-over parts in instantaneous frequency domain. The worst case, $M_{\mathrm{I}}=N(N-1) / 2$, is when the order of all IMFs is reversed, i.e. when the first IMF is under all others and the last IMF is above all others. However, this theoretical upper limit is very unlikely and the corresponding IMF set could be still considered upon index reversal.

\section{B. Validation method II}

Another validating measure is based on the Bedrosian theorem [14]. It refers to the necessary conditions for the signal's amplitude, $a(t)$, and phase, $\phi(t)$, to be exactly recoverable using Hilbert transformation. For signal $s(t)=a(t) \cos (\phi(t))$ these conditions require that the support of amplitude and phase's Fourier spectra are not overlapping. In other words, if the amplitude function, $f(t)=a(t)$, and the phase function, $g(t)=\cos (\phi(t))$, then

$$
\langle\mathcal{F}(f), \mathcal{F}(g)\rangle=0,
$$

where $\mathcal{F}$ represents the Fourier transform and $\langle h(t), l(t)\rangle=$ $\int h^{*}(t) l(t) d t$ is the dot product. Here it is assumed, that all functions belong to $L^{2}$ normed space. 


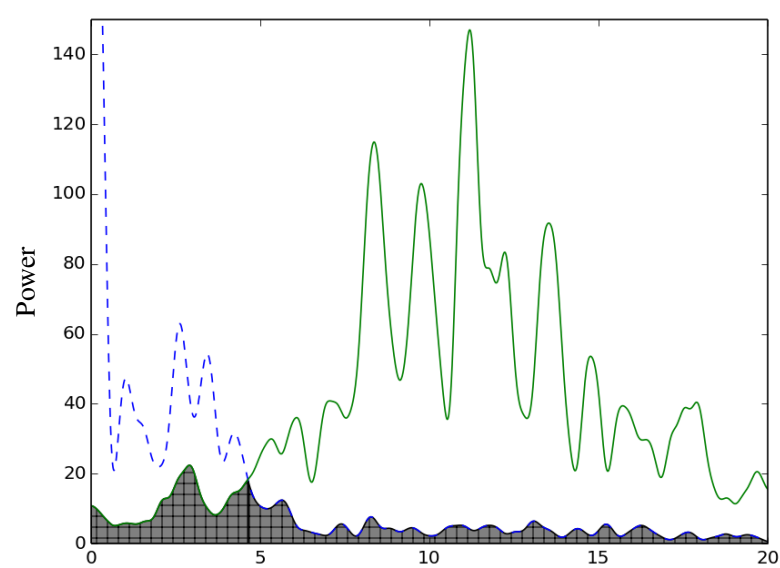

Frequency $[\mathrm{Hz}]$

Fig. 2. Example of comparing Fourier spectrum of the amplitude with the spectrum of phase. Gray-striped area indicates where two functions overlap.

Let $F_{j}^{a}=\left|\mathcal{F}\left(a_{j}(t)\right)\right|$ and $F_{j}^{\phi}=\left|\mathcal{F}\left(\cos \left(\phi_{j}(t)\right)\right)\right|$ be absolute values of Fourier transforms of $a_{j}$ and $\cos \left(\phi_{j}\right)$, respectively, for $j$ IMF. Their normalised measure of overlapping spectra is given as

$$
m_{j}^{\mathrm{II}}=\frac{\left\langle F_{j}^{a}, F_{j}^{\phi}\right\rangle}{\sqrt{\left\|F_{j}^{a}\right\|\left\|F_{j}^{\phi}\right\|}},
$$

where $\|h\|=\langle h, h\rangle$ is a norm of a function $h$. Assumptions of Bedrosian's theorem are completely fulfilled when spectra are not overlapping, thus minimum value of $m_{j}^{\mathrm{II}}$ is zero. This allows for different definitions of a metric for the whole IMF set, depending on application of EMD. First proposition is based on a biggest value of overlap $m_{j}$ in considered decomposition, i.e.

$$
M_{\mathrm{II}}=\max _{j}\left\{m_{j}^{\mathrm{II}}\right\}, \quad M_{\mathrm{II}} \in[0,1],
$$

and the second refers to the cumulative overlap within the decomposed set, i.e.

$$
M_{\mathrm{III}}=\sum_{j=1}^{N} m_{j}^{\mathrm{II}}, \quad M_{\mathrm{III}} \in[0, N],
$$

where in both cases $N$ is the number of extracted IMFs. Zero for both metrics implies no overlap between amplitude's and phase's spectra in any of IMFs.

Visual interpretation of the validation measure (6) is presented in Figure 2. It shows example Fourier spectra of slowly changing amplitude (dashed line) and higher frequency phase (solid line). Gray-striped region indicates overlapping area of both spectra. Proposed value is a measure of ratio of the overlapping area to the total area under both functions.

Since metric $M_{\text {III }}$ is a sum over all IMFs, it also contains the one which maximizes value $m_{j}^{\mathrm{II}}$ (Eq. (6)). This means that $M_{\text {III }}$ for each decomposition has to be at least equal or higher than $M_{\mathrm{II}}$.

\section{Application of the validation measures}

Each of the presented metrics highlights different properties of the decomposition. Computing all three values is equivalent to finding a point $M=\left(M_{\mathrm{I}}, M_{\mathrm{II}}, M_{\mathrm{III}}\right)$ in a 3-dimensional space, where each dimension relates to the specific metric. The best decomposition corresponds to the minimum over all the metrics, i.e. $M=(0,0,0)$, and the worst decomposition to $M=\left(\frac{N(N-1)}{2}, 1, N\right)$. For any other point one has to decide on the importance, or weight, for each of the proposed metrics, on the basis of the problem being considered. Although the distance in the $M$-space is not strictly defined, it can be any $L^{p}$ norm, thus we suggest using the weighted Manhattah metric, i.e.

$$
\|M\|=w_{1} M_{\mathrm{I}}+w_{2} M_{\mathrm{II}}+w_{3} M_{\mathrm{III}},
$$

where $w_{i}$ are respective weights. Their values should reflect the relative importance of features one is concentrated on.

\section{EXPERIMENT}

Measures proposed in section III quantify characteristics of well behaved IMFs. The smaller those metrics are, the better the IMF decomposition set represents the desired properties of the EMD-based decomposition.

One could also extend the EMD method into an optimization problem for any parameter, where cost is defined by $M_{i}$ measures. In the following examples, optimization was performed to choose the best value of a parameter FIXE_H for an input signal. The parameter FIXE_H indicates when to stop sifting procedure; it refers to the number of consecutive iterations of sifting for which the residue fulfils definition of an IMF. EMD was performed for each value of the parameter and the decomposition which minimized its value was chosen as the best.

For illustration two experiments were conducted. First one is performed on synthetic signal constructed of four sinusoidal components and the second on filtered Gaussian noise. All signals utilized in the following experiments, were generated with a single floating point precision. As it was shown in [15], in most cases this does not influence quality of the decomposition, but can increase the performance. Boundary effect introduced by using Hilbert transform was removed by symmetrically truncating the signal to $80 \%$ of the original, i.e. removing initial and final $10 \%$ of samples. Additionally, the signal was smoothed by adding mean of each sample's neighbours.

\section{A. Experiment 1}

First experiment was conducted on a synthetic signal composed of harmonic components. The test signal was generated according to the following formula

$$
S_{1}(t)=\sum_{j=1}^{5} A_{j} \sin \left(2 \pi f_{j} t+\phi_{j}\right)+\mathcal{N}(0,0.1),
$$

where values for the amplitude $\left(A_{j}\right)$, the frequency $\left(f_{j}\right)$ and the phase shift $\left(\phi_{j}\right)$ are included in Table I. The values of these parameters were selected at random, with constrains 


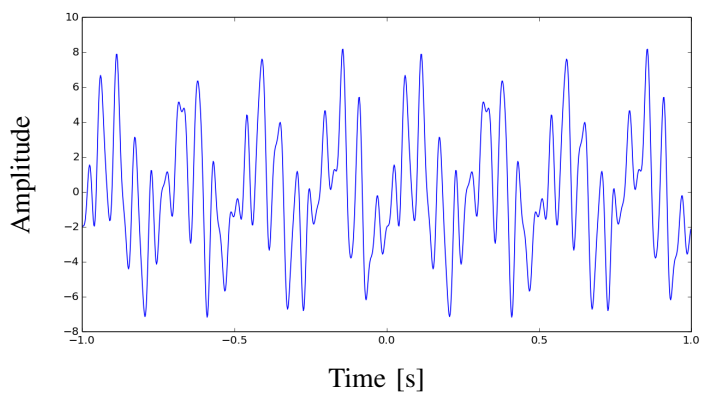

Fig. 3. Test signal $S_{1}(t)$ used in experiment with synthetic data, generated according to Eq. (10).

that amplitude had to be an integer smaller than 5 and that minimum distance between frequencies was 4 . Moreover, in equation (10) the symbol $\mathcal{N}(\mu, \sigma)$ denotes noise in the form of the normal distribution with a mean $\mu$ and a variance $\sigma^{2}$. The graphical representation of the signal can be seen in Fig. 3.

The experiment was conducted as follows:

1) Generate test signal $S_{1}(t)$.

2) Set value range of the parameter - FIXE_H spanning from 0 to 20.

3) For each value FIXE_H, decompose the signal with EMD and calculate all metrics $\left(M_{\mathrm{I}}, M_{\mathrm{II}}\right.$ and $\left.M_{\mathrm{III}}\right)$.

4) The best decomposition set is the one with the smallest sum of all metrics $M=M_{\mathrm{I}}+M_{\mathrm{II}}+M_{\mathrm{III}}$ (all weights equal).

TABLE I

PARAMETERS FOR AMPLITUDE $\left(A_{j}\right)$, FREQUENCY $\left(f_{j}\right)$ AND PHASE SHIFT $\left(\phi_{j}\right)$ OF EQUATION 10.

\begin{tabular}{|c|ccc|}
\hline$j$ & $A_{j}$ & $f_{j}$ & $\phi_{j}$ \\
\hline 1 & 1 & 35 & 2.0 \\
2 & 1 & 25 & 4.0 \\
3 & 3 & 19 & 0.0 \\
4 & 2 & 15 & 3.4 \\
5 & 3 & 4 & 5.7 \\
\hline
\end{tabular}

The signal was decomposed 20 times with the EMD under different stopping criteria conditions, i.e. FIXE_H ranged from 1 to 20. All validating metrics, computed for each decomposition, are presented in the Table II. Decomposition sets are assessed based on a sum total of all metric values for a parameter. The best set is the one with the smallest sum; likewise the worst set is one with the largest value.

For the provided signal, the best decomposition was obtained with the parameter FIXE_H $=17$. The figure 4 shows all components. Likewise, the worst decomposition set is obtained for FIXE_H $=1$ (Fig. 5). Although these results appear similar, especially when comparing the first IMFs of both decompositions, there are few differences between obtained sets. Main difference is in the number of produced components. The set with higher value of metric $M$ has one additional IMF. It might not be obvious at which stage it was added, but there seems to be more components with low number of extrema. Moreover, the difference is also clear when analysing position and amplitude of extrema. Comparing second and third IMFs one can see that in the best decomposition

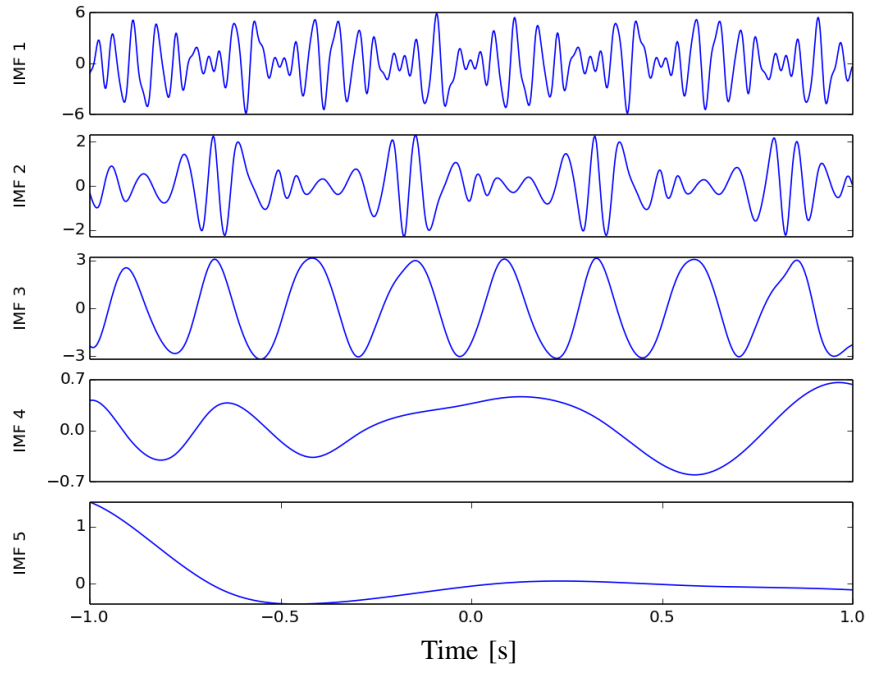

Fig. 4. The best EMD decomposition set, i.e. producing the smallest metric $M$ value, for given range of FIXE_H parameters. Decomposition obtained from signal $S_{1}(t)$.

position of local extrema are relatively evenly spaced. The same observation holds for the amplitude of extrema - there is a clear stable modulation in the amplitude. In contrast, the worst decomposition has less visible structure, even though it also contains an apparent repeating pattern.

TABLE II

METRIC VAlues OBTAINED BY PERFORMING THE EMD ON $S_{1}(t)$ WITH VARYING VALUE OF FIXE_H PARAMETER.

\begin{tabular}{|c|l|l|l||c|}
\hline FIXE_H & $M_{\text {I }}$ & $M_{\text {II }}$ & $M_{\text {III }}$ & $M_{\text {I }}+M_{\text {II }}+M_{\text {III }}$ \\
\hline 1 & 1.17 & 0.63 & 0.87 & 2.66 \\
2 & 1.13 & 0.57 & 0.77 & 2.47 \\
3 & 1.33 & 0.48 & 0.53 & 2.27 \\
4 & 1.17 & 0.61 & 0.88 & 2.65 \\
5 & 0.91 & 0.22 & 0.51 & 1.64 \\
6 & 0.92 & 0.22 & 0.51 & 1.65 \\
7 & 0.93 & 0.22 & 0.50 & 1.64 \\
8 & 1.15 & 0.35 & 0.69 & 2.19 \\
9 & 1.12 & 0.32 & 0.66 & 2.10 \\
10 & 1.12 & 0.35 & 0.72 & 2.19 \\
11 & 1.18 & 0.30 & 0.54 & 2.02 \\
12 & 1.20 & 0.29 & 0.54 & 2.03 \\
13 & 0.98 & 0.14 & 0.31 & 1.44 \\
14 & 0.98 & 0.16 & 0.32 & 1.45 \\
15 & 0.96 & 0.16 & 0.31 & 1.43 \\
16 & 0.97 & 0.16 & 0.32 & 1.45 \\
17 & 0.96 & 0.16 & 0.30 & 1.42 \\
18 & 1.44 & 0.48 & 0.64 & 2.56 \\
19 & 1.45 & 0.36 & 0.50 & 2.32 \\
20 & 1.56 & 0.27 & 0.45 & 2.24 \\
\hline
\end{tabular}

\section{B. Experiment 2}

For this experiment signal was generated using Gaussian noise with mean value 0 and standard deviation 1

$$
S_{2}(t)=\mathcal{N}(0,1) .
$$

As it has been suggested in [10], in order to obtain meaningful decomposition it is necessary to have ratio of signal's sampling frequency, $f_{s}$, to the highest Fourier frequency, $f_{f}$, of at least $10\left(f_{s} / f_{f} \geq 10\right)$. This means that when sampling with 


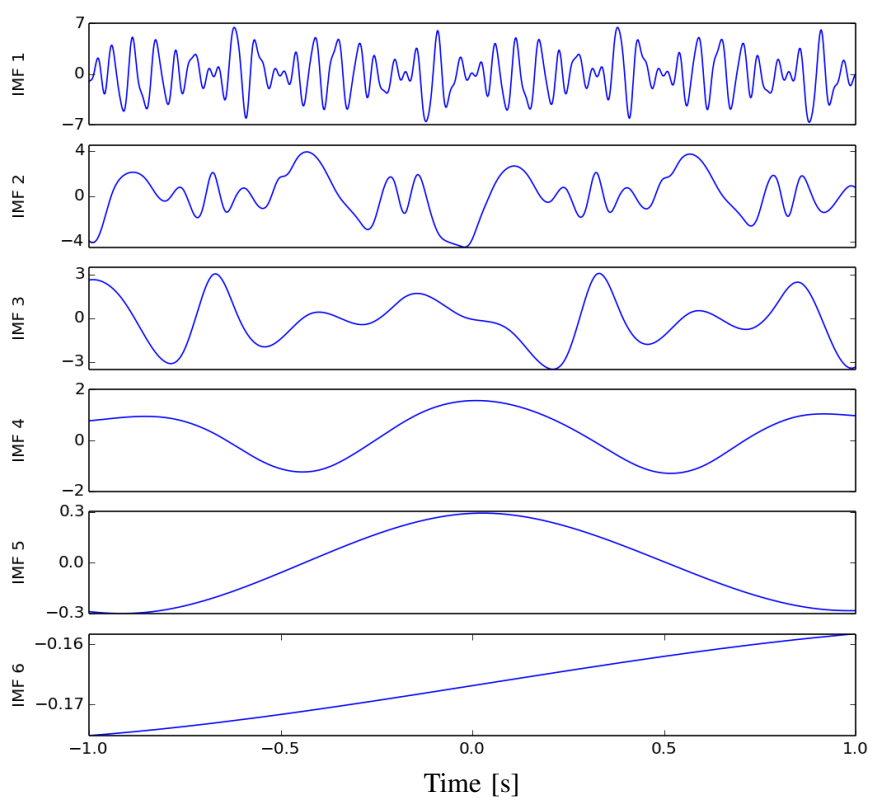

Fig. 5. The worst EMD decomposition set, i.e. producing the biggest metric $M$ value, for given range of FIXE_H parameters. Decomposition obtained from signal $S_{1}(t)$.

frequency $500 \mathrm{~Hz}$, signal has to be low-pass filtered with the cut off frequency of $50 \mathrm{~Hz}$. For the experiment, we used the zero-phase Butterworth low-pass filter of order 4 . The resulting signal is visualised in Figure 6.

The experiment was conducted similarly to the Experiment I, with the difference in weights used to calculate the metric. The exact steps of execution were:

1) Generate the test signal, $S_{2}(t)$.

2) Set the value range of the parameter - FIXE_H spanning from 1 to 20.

3) For each value FIXE_H, decompose the signal with the EMD and calculate all metrics $\left(M_{\mathrm{I}}, M_{\mathrm{II}}\right.$ and $\left.M_{\mathrm{III}}\right)$.

4) The best decomposition set is the one with the smallest sum of all metrics $M=2 \cdot M_{\mathrm{I}}+M_{\mathrm{II}}+0.5 \cdot M_{\mathrm{III}}$.

Such a choice of weights puts more emphasis on selecting IMFs with more mutually separate instantaneous frequencies. It also increases significance of the component with the most overlapping amplitude and phase Fourier spectra; the sum of all measuring values (Eq. (6)) has lower priority. Overall, the metric, $M$, is meant to select a decomposition with the most distinct frequencies. This effect should be visible when analysing location of extrema, as they should be spaced more evenly.

All calculated values of metrics are presented in the Table III. The last column contains a weighted sum of all other metrics for each value of the parameter FIXE_H. The smallest and the largest values are obtained for FIXE_H equal to 2 and 12, respectively. The best decomposition can be seen in the Figure 7, whereas the worst in the Figure 8. Comparing the two decompositions one can see the difference in the number of IMFs - two more in the worst EMD set. In this case, two first IMFs seem to be similar, or at least without any obvious differences. Analysing position and value of the

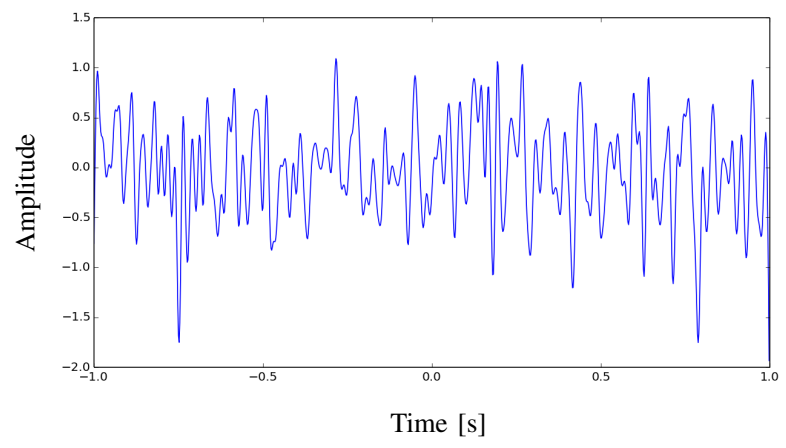

Fig. 6. Test signal $S_{2}(t)$ used in experiment with filtered Gaussian noise generated.

third component's extrema, one can see that there are more extrema for FIXE_H $=12$. Additionally, IMF 6 from the worst decomposition does not seem to have any close counterpart in the best decomposition. Its small amplitude suggests that the component might be hidden within amplitude modulation of any other IMF.

TABLE III

METRic VALUES OBTAINED BY PERFORMING THE EMD ON $S_{2}(t)$ With VARYING VALUE OF FIXE_H PARAMETER.

\begin{tabular}{|c||c|c|c||c|}
\hline FIXE_H & $M_{\text {I }}$ & $M_{\text {II }}$ & $M_{\text {III }}$ & $2 M_{\text {I }}+M_{\text {II }}+0.5 M_{\text {III }}$ \\
\hline 1 & 1.64 & 0.60 & 0.84 & 4.30 \\
2 & 1.62 & 0.59 & 0.70 & 4.18 \\
3 & 1.78 & 0.58 & 0.85 & 4.56 \\
4 & 2.17 & 0.56 & 0.67 & 5.23 \\
5 & 2.27 & 0.53 & 0.82 & 5.48 \\
6 & 2.17 & 0.45 & 0.69 & 5.14 \\
7 & 2.25 & 0.47 & 0.79 & 5.37 \\
8 & 2.07 & 0.57 & 0.89 & 5.15 \\
9 & 2.21 & 0.53 & 1.04 & 5.47 \\
10 & 2.20 & 0.55 & 0.86 & 5.38 \\
11 & 2.25 & 0.55 & 0.87 & 5.48 \\
12 & 2.58 & 0.60 & 1.04 & 6.28 \\
13 & 2.56 & 0.60 & 1.00 & 6.22 \\
14 & 2.17 & 0.51 & 0.74 & 5.22 \\
15 & 2.54 & 0.61 & 0.81 & 6.09 \\
16 & 2.51 & 0.60 & 0.80 & 6.02 \\
17 & 2.52 & 0.60 & 0.80 & 6.04 \\
18 & 2.51 & 0.60 & 0.78 & 6.01 \\
19 & 2.52 & 0.60 & 0.78 & 6.03 \\
20 & 2.59 & 0.58 & 0.99 & 6.26 \\
\hline
\end{tabular}

\section{Conclusion}

In the recent years, EMD has been proven very useful in many areas of science and engineering. Unfortunately, due to its flexibility and a lack of mathematical framework, this technique has been misused. Metrics proposed in this paper refer to idealized characteristics of EMD - either suggested, or intended while creating the method. In contrast to the most validation methods discussed in the literature, the ones proposed in this paper are objective, independent from user's subjective judgment of the decomposition. Since EMD creates a set of oscillatory components, the properties on which these metrics were based are related to their frequencies. Validation of the whole IMF decomposition set is performed either on the basis of significant and mutually separate instantaneous 


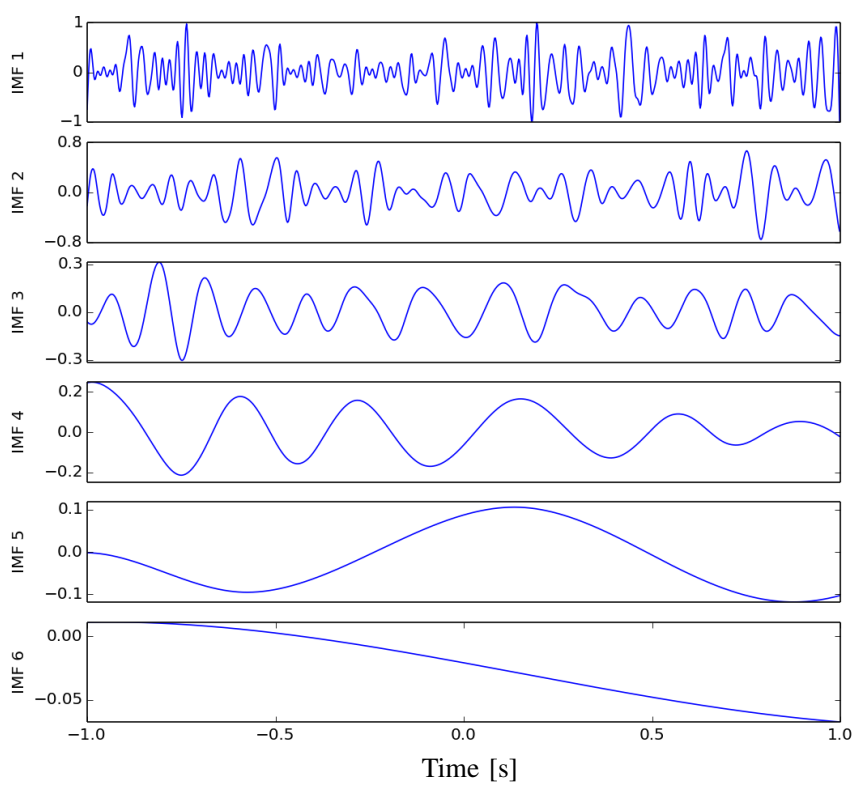

Fig. 7. The best EMD decomposition set, i.e. producing the smallest metric $M$ value, for given range of FIXE_H parameters. Decomposition obtained from signal $S_{2}(t)$

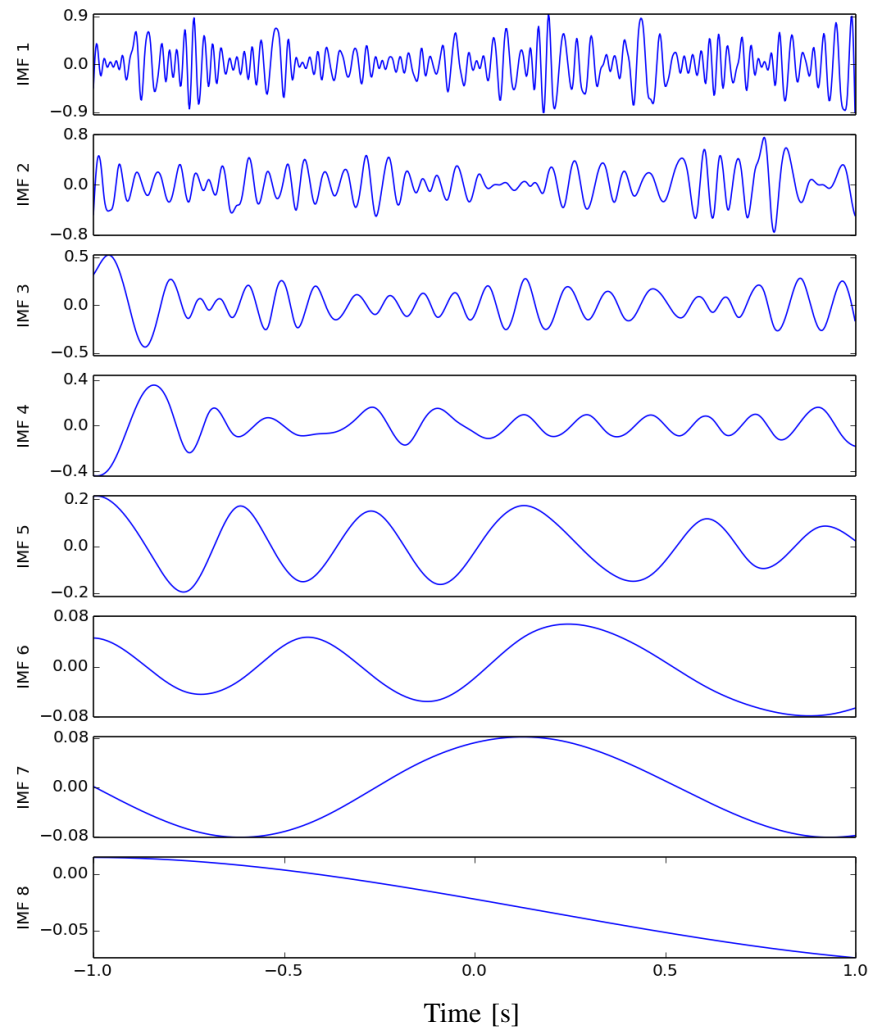

Fig. 8. The worst EMD decomposition set, i.e. producing the biggest metric $M$ value, for given range of FIXE_H parameters. Decomposition obtained from signal $S_{2}(t)$. frequencies, or on the attempt to fulfill, or be closer to, the Bedrosian conditions.

An analysis of examples presented in the section IV seems to support the usefulness of the proposed metrics. Although the behaviour of the decomposition can be changed by adjusting weights $w_{i}$ of the desired feature (Eq. (9)), it should still provide good results. The best decomposition always produces less IMFs, which suggests having more compact information representation of the original signal. Moreover, visual inspection confirms that the best decompositions have better structured (evenly spaced locations of extrema) than the worst ones.

Nevertheless, despite the foregoing discussion, until there is a mathematical foundation of the EMD, it is impossible to create a single metric, which would capture all required features. Proposed measures should be considered as an assistance for an inexperienced user, providing him with additional arguments for used parameters choices.

\section{REFERENCES}

[1] N. E. Huang, Z. Shen, S. R. Long, M. C. Wu, H. H. Shih, Q. Zheng, N.C. Yen, C. C. Tung, and H. H. Liu, "The empirical mode decomposition and the Hilbert spectrum for nonlinear and non-stationary time series analysis," 1998.

[2] N. E. Huang and Z. Wu, "A review on Hilbert-Huang transform: Method and its applications to geophysical studies," 2008.

[3] T. M. Rutkowski, D. P. Mandic, A. Cichocki, and A. W. Przybyszewski, "EMD approach to multichannel EEG data - the amplitude and phase components clustering analysis," 2010.

[4] D. Mandic, N. Rehman, Z. Wu, and N. Huang, "Empirical Mode Decomposition-Based Time-Frequency Analysis of Multivariate Signals: The Power of Adaptive Data Analysis," Signal Processing Magazine, IEEE, vol. 30, no. 6, pp. 74-86, Nov 2013.

[5] Z. Wu and N. E. Huang, "Ensemble Empirical Mode Decomposition: a Noise-assisted Data Analysis Method," Advances in Adaptive Data Analysis, vol. 1, no. 1, pp. 1-41, jan 2009. [Online]. Available: http://www.worldscientific.com/doi/abs/10.1142/S1793536909000047

[6] S. Meignen and V. Perrier, "A New Formulation for Empirical Mode Decomposition Based on Constrained Optimization," IEEE Signal Processing Letters, vol. 14, no. 12, pp. 932-935, dec 2007.

[7] C. Huang, L. Yang, and Y. Wang, "Convergence of a ConvolutionFiltering-Based Algorithm for Empirical Mode Decomposition," Advances in Adaptive Data Analysis, vol. 01, no. 04, pp. 561-571, oct 2009.

[8] B. Matei and S. Meignen, "A new optimization based approach to the empirical mode decomposition," Annals of the University of Bucharest, vol. 1, no. 4, pp. 129-139, 2014

[9] S. Riemenschneider, B. Liu, Y. Xu, and N. E. Huang, "B-spline based empirical mode decomposition," 2005.

[10] N. Stevenson, M. Mesbah, and B. Boashash, "A sampling limit for the empirical mode decomposition," in ISSPA 2005: The 8th International Symposium on Signal Processing and its Applications, Vols 1 and 2. Proceedings, vol. 2. IEEE, 2005, pp. 647-650.

[11] S. Qin and Y. Zhong, "A new envelope algorithm of HilbertHuang Transform," Mechanical Systems and Signal Processing, vol. 20, no. 8, pp. 1941-1952, 2006. [Online]. Available: http://www.sciencedirect.com/science/article/pii/S0888327005000993

[12] R. Rato, M. Ortigueira, and A. Batista, "On the HHT, its problems, and some solutions," Mechanical Systems and Signal Processing, vol. 22, no. 6, pp. 1374 1394, 2008, special Issue: Mechatronics. [Online]. Available: http://www.sciencedirect.com/science/article/pii/S0888327007002701

[13] L. Cohen, Time-frequency Analysis, 1995.

[14] E. Bedrosian, "A product theorem for Hilbert transforms," Proceedings of the IEEE, vol. 51, no. 5, pp. 868-869, May 1963.

[15] D. Laszuk, O. Cadenas, and S. J. Nasuto, "EMD performance comparison: Single vs Double floating points," International Journal of Signal Processing Systems, vol. 4, no. 4, pp. 349-353, 2016. 


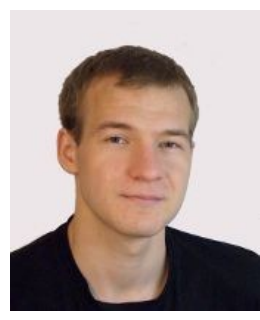

Dawid Laszuk has received his MSc in Physics (Biomedical specialty) from the University of Warsaw. His thesis concentrated on creating interface and data analysis methods for hybrid brain computer interfacing. He is currently engaged in research to achieve his $\mathrm{PhD}$ in Electronic Engineering; this work involves empirical mode decomposition implemented on a graphical processing unit for the analysis of neurological signals (primarily EEG). His research interests also include augmentation and integration of organic and electrical components.

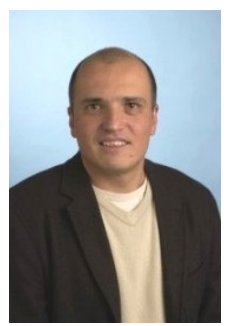

Oswaldo Cadenas received a $\mathrm{PhD}$ in Computer science from the University of Reading in 2002 where he has remained as lecturer. Dr Cadenas works on parallel formulation of algorithms and its implementation as parallel hardware architectures.

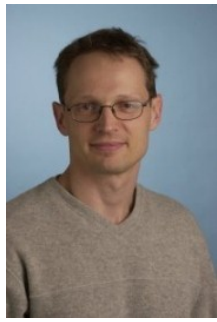

Sławomir J Nasuto has received an MSc in Mathematics from the University of Marie CurieSklodowska in Lublin, Poland in 1993 and a PhD in Cybernetics from University of Reading in 1999. He has been a member of academic staff in School of Systems Engineering at Reading since 2000. His research interests span computational neuroscience and neuroanatomy, analysis of signals generated by the nervous system including EEG, single neuron and multivariate spike trains or EMG and their applications for Brain Computer Interfaces and Animats (robots controlled by neural cultures). He also has research interests in Stochastic Diffusion Search, an algorithm belonging to a family of Swarm Intelligence methods and he has been involved in applications of such techniques in aerospace problems. 